\title{
APLICAÇÃO DO MÉTODO DMAIC NA MELHORIA DO PROCESSO DE GERENCIAMENTO DE FORNECEDORES EM UMA EMPRESA DO SETOR DE ÓLEO E GÁS
}

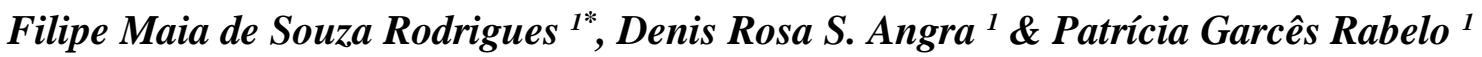

\begin{abstract}
RESUMO
RODRIGUES, F. M. S.; ANGRA, D. R. S. ; RABELO, P. G. Aplicação da metodologia lean six sigma ao gerenciamento de fornecedores de uma empresa do setor de óleo e gás. Perspectivas Online: Exatas \& Engenharia, v. 10, n. 28, p. 1-17, 2020.
\end{abstract}

Em qualquer tipo de indústria a busca por maior eficiência e melhores resultados é constante. No mercado de óleo e gás a pressão sofrida pelas empresas é ainda maior. As altas cifras movimentadas, as constantes oscilações e as crises do mercado tornam o setor um dos mais desafiadores para se operar. Nesse contexto, as exigências por redução de custos, tempos de processamento e desperdícios cresce continuamente, tornando o gerenciamento da cadeia de suprimentos um dos pontos estratégicos na busca por vantagens competitivas. Ao gerenciar qualquer cadeia de suprimentos o desempenho dos fornecedores é primordial. Uma organização que não gerencia de perto sua rede de fornecedores não alcançará os benefícios inerentes a essa prática e ficará certamente um passo atrás de seus concorrentes. $\mathrm{O}$ objetivo deste trabalho é a melhoria no processo de gerenciamento de fornecedores de uma empresa do setor de óleo e gás por meio da aplicação da metodologia lean. Por intermédio da aplicação do método DMAIC foram identificados e tratados pontos de melhoria no sistema, buscando eliminar focos de desperdício e aumentar a eficiência do processo. Como resultado, houve uma redução do número de fornecedores, tornando seu gerenciamento mais eficiente; obteve-se um menor lead time total na compra de materiais; diminuição do número de atrasos na entrega e no número de não conformidades identificadas. $\mathrm{O}$ método DMAIC se mostrou eficaz ao ser aplicado na cadeia de suprimentos, melhorando a gestão de fornecedores da companhia e trazendo um melhor desempenho para o processo.

Palavras-chave: Gestão de fornecedores; DMAIC; Metodologia Lean Six Sigma

\footnotetext{
${ }^{1}$ Universidade Federal Fluminense - UFF - Instituto de Ciência e Tecnologia - ICT - Campus Rio das Ostras, Rua Recife, s/n, Jardim Bela Vista, Rio das Ostras, RJ, CEP: 28895-532, Brasil;

(*) e-mail: frodrigues@id.uff.br
} 


\title{
APPLICATION OF DMAIC METHOD FOR IMPROVEMENT OF SUPPLIERS MANAGEMENT PROCESS IN AN OIL AND GAS COMPANY
}

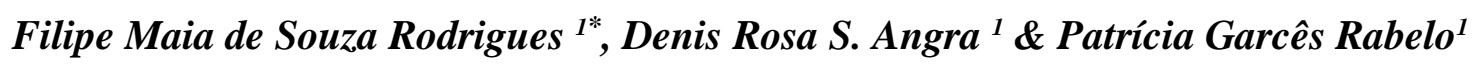

\begin{abstract}
In industry, the search for greater efficiency and better results is constant. In oil and gas market, the pressure suffered by companies is even greater. The amount of money involved, the constant market oscillations and crises make the sector one of the most challenging to operate. In this context, the demands for costs, processing times and waste reductions increase every moment, making supply chain management one of the strategic points in the search for competitive advantages. When managing any supply chain, supplier performance is paramount. An organization that does not manage closely its suppliers' network will not achieve the benefits inherent in this practice and will be lag behind its
\end{abstract}

ABSTRACT

RODRIGUES, F. M. S.; ANGRA, D. R. S. ; RABELO, P. G. Application of DMAIC method for improvement of suppliers management process in an oil and gas company (In Portuguese). Perspectivas Online: Exatas \& Engenharia, v. 10, n. 28, p. 1-17, 2020.

competitors. The objective of this paper is to apply lean methodology to supplier management on an oil and gas company supply chain. Improvements opportunities were identified and treated through the application of the DMAIC, trying to eliminate waste points and increase the process efficiency. As a result, there was a reduction in the number of suppliers, allowing a more efficient management; a shorter lead time was obtained in the purchase of materials; decrease in the number of delayed deliveries and in the number of non-conformities identified. The DMAIC method has proven effective when applied to the supply chain, improving the company's supplier management and bringing better performance to the process.

Keywords: Suppliers Management; DMAIC; Lean Six Sigma Methodology.

\footnotetext{
${ }^{1}$ Northern Fluminense State University Darcy Ribeiro - UFF - Science and Technology Institute - ICT -Rio das Ostras Campus, Street Recife, w/n, Jardim Bela Vista, Rio das Ostras, RJ, Postal code: 28895-532, Brazil; $(*)$ e-mail: frodrigues@id.uff.br
} 


\section{INTRODUÇÃO}

No passado, a relação entre as empresas e seus consumidores era completamente diferente do que se vê hoje. Não existiam cadeias de suprimentos desenvolvidas que permitissem os produtos serem levados do seu ponto de produção aos pontos de consumo de forma rápida e eficiente. A globalização e os avanços da tecnologia permitiram que os mercados mundiais chegassem ao nível de integração em que estão no momento. As organizações perceberam os benefícios advindos de uma cadeia de suprimentos eficiente e passaram a investir no tema. Atualmente, a cadeia de suprimentos é parte do processo de geração de valor para o consumidor final, tendo um papel fundamental no planejamento estratégico das grandes organizações (LUMMUS e VOKURKA, 1999; USUI et al., 2017).

O conceito de cadeia de suprimentos, que foi construído ao longo dos anos, pode ser resumido como "múltiplas empresas colaborando para incrementar seus posicionamentos estratégicos e melhorar suas eficiências operacionais" (BOWERSOX et al, 2013). O gerenciamento da cadeia de suprimentos, ou SCM da sigla em inglês, envolve tanto o fluxo de materiais como de informações ao longo das organizações inseridas no processo (SALAH, 2011).

Brito e Berardi (2010) mostram que, se por um lado, a geração de valor nas relações organizacionais depende de aspectos como colaboração entre as partes, que vão além das relações de controle, extrapolando para a criação de ativos; por outro lado, o comportamento colaborativo exige relações de confiança que superem o receio e a imposição de proteções processuais. Clássicos exemplos são dados pelo estudo do relacionamento das montadoras japonesas e seus fornecedores na criação de ativos específicos e cooperação (DYER, 1996).

Os mesmos autores discutem como a relação entre parceiros comerciais também pode ser marcada pela definição de padrões mínimos de eficiência, por procedimentos de controle e pela exclusão de fornecedores, ou por formas cooperativas de desenvolvimento. E demonstram como a pressão externa seja regulatória, do mercado ou da sociedade, afeta a relação com a cadeia de fornecedores, seguido por fatores como objetivos de melhoria do desempenho econômico e ambiental (BRITO e BERARDI, 2010).

Segundo Martins (2011), a vantagem competitiva de uma empresa, qualquer que seja o setor em que se insere, está diretamente ligada à relação com os membros de sua supply chain. Organizações com cadeias de suprimentos mais eficientes terão clara vantagem sobre suas concorrentes. De acordo com Scholten e Schilder (2015), a colaboração entre empresas se tornou fundamental para o desenvolvimento de vantagens competitivas como velocidade de resposta e flexibilidade.

Hoinaski (2016) afirma que uma organização que hoje não dá a devida atenção à seleção e gerenciamento de seus fornecedores terá dificuldades em obter vantagens competitivas frente aos concorrentes. Nesse contexto, é nítido que o relacionamento entre cliente e fornecedor é a chave para a eficiência do processo de suprimentos e, consequentemente, para o sucesso das empresas dos mais diversos tipos de mercado. Com o passar dos anos, o gerenciamento de fornecedores se tornou uma ferramenta fundamental (KANNAN e TAN, 2002).

Kannan e Tan (2002) discorrem ainda a respeito de diferentes aspectos da gestão de fornecedores, sendo elas a seleção, o desenvolvimento e a avaliação de fornecedores. Estas podem ser consideradas etapas de um processo global que tem como principal objetivo o

Persp. Online: exatas \& eng., Campos dos Goytacazes, 28 (10) 1 - 17 - 2020

https://ojs3.perspectivasonline.com.br/ 
aumento da eficiência da cadeia de suprimentos.

Para que tal objetivo seja alcançado, existem diversas fontes que podem contribuir, a fim de que a empresa que a persegue consiga desenvolver o gerenciamento de seus fornecedores. Técnicas de qualidade são por vezes aplicadas. Algumas ferramentas baseadas em cooperação entre empresas e outras que promovem a competição também são utilizadas (PARK et al, 2010). Em adição a tais métodos, nota-se recentemente nessa área, assim como em outros segmentos, a influência da metodologia lean. O lean manufacturing tem como objetivo a melhoria da qualidade, eliminação de desperdícios, redução do lead time e do custo total do processo. O gerenciamento da cadeia de suprimentos carece de metodologias que deem esse suporte analítico (SALAH, 2011).

Nessa direção, em diversos trabalhos recentes as ferramentas do método Six Sigma para redução de variabilidade têm sido incorporadas ao lean manufacturing. O termo Lean Six Sigma é hoje amplamente utilizado (ALBLIWI, ANTONY e LIM, 2015; SALAH, RAHIM e CARRETERO, 2010). Dhafer A. e Aramco S. (2012) dizem que o termo Lean Six Sigma representa uma combinação poderosa que alcança processos otimizados e um gerenciamento de negócios eficaz. O conceito Lean Six Sigma pode ser usado como modelo de melhoria de desempenho, baseado no ciclo PDCA, sob o nome DMAIC (Define-MeasureAnalyze-Improve-Control). Ainda segundo os autores, este modelo de melhoria e redesenho de processos permite alcançar desempenhos de processos Six Sigma.

Para Dreachslin e Lee (2007), Bargerstock e Richards (2015), o ciclo de melhoria usando o método DMAIC consiste nos seguintes elementos:

- Definir $\rightarrow$ nesta etapa deve-se identificar os seguintes elementos: problema (descrição do problema, tempo de ocorrência), escopo do projeto (elementos do processo em que a equipe trabalhará) e objetivo do projeto (um objetivo tangível de alcançar e sustentar no futuro);

- Medir $\rightarrow$ durante o estágio de medição, os parâmetros e locais de medição devem ser definidos, ou seja, os pontos de qualidade do processo e seus custos, juntamente com um reflexo preciso do estado atual. Realizar medições com sucesso requer uma perspectiva estatística sobre os processos de produção específicos e problemas relacionados a eles;

- Analisar $\rightarrow$ durante esta fase da metodologia, analisando os parâmetros do processo, a equipe será capaz de determinar as causas do problema, que precisará ser eliminado ou corrigido. Os resultados obtidos durante a etapa de medição são utilizados para investigar a correlação entre causas de defeitos e fontes de variabilidade de processo;

- Melhorar $\rightarrow$ consiste em procurar e avaliar as possíveis causas da variabilidade e inconsistências do processo e investigar suas correlações. Aprender as relações multifatoriais permite alcançar os resultados desejados;

- Controlar $\rightarrow$ o estágio de controle ocorre após a conclusão da nova fase de implementação do processo. O objetivo fundamental é a observação constante das melhorias introduzidas para manter um nível desejado de qualidade. Nesta fase do DMAIC, o sistema de medição e o processo de verificação de potencial são repetidos para confirmar a melhoria do processo (REHMAN et al., 2018).

Persp. Online: exatas \& eng., Campos dos Goytacazes, 28 (10) 1 - $17-2020$ 
Segundo Sokovic et al. (2010), o DMAIC nasceu do Six Sigma como uma "abordagem cíclica orientada por dados para melhoria de processos". Atualmente sua aplicação já vai além da esfera industrial em que foi criado, podendo ser adaptado a praticamente qualquer processo de melhoria, inclusive áreas de gestão e cadeia de suprimentos (FIRAT et al., 2017).

O presente trabalho foi desenvolvido em uma grande empresa que atua no mercado de óleo e gás, mais especificamente na perfuração de poços offshore. A direção da empresa entende que havia oportunidades de melhoria na área de suprimentos, especialmente em decorrência de dois problemas recorrentes: o alto índice de atraso na entrega de materiais e o elevado número de não conformidades nesse processo.

A partir dessa necessidade, foram estabelecidos como objetivos da pesquisa a redução do percentual de materiais entregues em atraso e do número de não conformidades no processo de suprimentos, por meio da utilização da metodologia Lean Six Sigma e, mais especificamente, de uma de suas ferramentas: o método DMAIC.

\section{METODOLOGIA}

A pesquisa possui características qualitativas e quantitativas em sua abordagem. Nas etapas de análise do problema e mapeamento dos processos nota-se um viés qualitativo, pois busca-se compreender a totalidade do fenômeno com intuito de produzir informações relevantes a serem utilizadas em outros momentos do trabalho. Já durante a coleta e análise de dados numéricos e cálculo de indicadores de desempenho, a pesquisa assume um caráter quantitativo, recorrendo a ferramentas matemáticas e estatísticas para se estabelecer relações entre variáveis e resultados das ações implementadas (FONSECA, 2002; GIL, 2002)

Quanto à natureza, pode-se definir a pesquisa como aplicada, uma vez que tem como objetivo gerar conhecimentos de aplicação prática, voltados para resolver problemas específicos em determinado cenário (SILVEIRA e CÓRDOVA, 2009).

Com relação aos objetivos, o trabalho possui cunho exploratório. Esse tipo de pesquisa é utilizado para aprofundar o conhecimento em determinado problema e construir hipóteses relacionadas à situação em estudo. Normalmente envolve uma pesquisa bibliográfica sobre o assunto, seguida de uma análise do sistema por meio de observações ou entrevistas, e finalmente a formulação de hipóteses envolvendo o problema em questão (GIL, 2002).

A pesquisa é definida, com relação aos procedimentos, como uma pesquisa-ação. De acordo com Dresch, Lacerda e Júnior (2015, p. 25) a pesquisa-ação "tem como objetivo resolver ou explicar problemas encontrados em certo sistema. (...) Na pesquisa ação o pesquisador deixa de ser um observador e passa a ter um papel ativo na investigação; ele contribui e interage com o objeto de estudo". Ainda segundo os autores esse tipo de pesquisa geralmente engloba as etapas listadas a seguir, seguidas ao longo deste trabalho. Nota-se que existe forte correspondência às etapas do DMAIC, citadas anteriormente:

1. Coletar os dados necessários

2. Analisar os dados obtidos

3. Planejar as ações a serem implementadas

4. Implementar as ações

5. Avaliar resultados obtidos

Persp. Online: exatas \& eng., Campos dos Goytacazes, 28 (10) 1 - 17 - 2020

https://ojs3.perspectivasonline.com.br/ 
Divide-se a metodologia utilizada da seguinte forma: em um primeiro momento, com objetivo de adquirir um conhecimento mais amplo sobre o assunto, foi realizada uma revisão da literatura englobando os conceitos que seriam utilizados, dentre os quais os principais são gerenciamento de fornecedores na supply chain e o lean six sigma e suas ferramentas. $\mathrm{Na}$ segunda parte do estudo foi feita uma avaliação do processo de gestão de fornecedores da empresa, por meio de reuniões com os profissionais e coleta de dados dos sistemas ERP, obtendo, dessa forma, um retrato do sistema que estava em vigor. Aplicou-se, na terceira parte da pesquisa, o método DMAIC, visando aprimorar o processo em questão.

O método DMAIC foi aplicado seguindo as fases descritas na seção anterior. Iniciouse com a definição do problema, estabelecendo-se qual o escopo seria coberto pela pesquisa. $\mathrm{Na}$ etapa de medição foram calculados indicadores de desempenho para o processo, obtendo um panorama antes de qualquer modificação. Rodrigues e Sellitto (2009) argumentam que indicadores são ferramentas à disposição das organizações para colocar em prática suas estratégias. Eles irão guiar os esforços da empresa para a melhoria de seus processos. A abordagem mais apropriada para se realizar a medição de desempenho de fornecedores é utilizar ambos os tipos de indicadores, qualitativos e quantitativos. A escolha desses indicadores irá depender da estratégia da organização (TAHERDOOST e BRARD, 2019).

Em seguida, os resultados dos indicadores foram analisados e foram traçadas estratégias para aprimorar pontos específicos do processo, colocadas em prática na fase seguinte. Por fim, como última etapa do DMAIC, foram medidos e acompanhados os resultados posteriores à pesquisa para verificar se os objetivos foram atingidos. $\mathrm{O}$ desenvolvimento dos passos descritos será detalhado no próximo tópico deste trabalho.

\section{RESULTADOS E DISCUSSÃO}

Nessa seção será detalhada a aplicação do método DMAIC na gestão da cadeia de suprimentos da organização. As etapas utilizadas seguem a ordem apresentada anteriormente: Definir, Medir, Analisar, Melhorar e Controlar.

\subsection{Definir}

A empresa em que foi desenvolvido o projeto passa atualmente por uma reestruturação de seus processos internos, buscando um aumento na eficiência e redução dos custos. A perseguição por processos mais enxutos e corte de desperdícios vem sendo uma tendência nos últimos anos.

A capacidade em estabelecer um relacionamento estreito e duradouro com parceiros estratégicos tornou-se fator crucial para alcançar vantagens competitivas. Segundo os autores Andersen e Skjoett-Larsen (2009), a cadeia de suprimentos passou de perspectivas operacionais (compras, qualidade e outros) para perspectivas estratégicas com foco na relação de longo prazo com parceiros. Dado este papel fundamental da cadeia de suprimentos no funcionamento das empresas atualmente, o setor se tornou um dos focos principais na busca por melhorias dentro das organizações.

A partir do entendimento que a gestão da cadeia de suprimentos deveria ganhar mais importância na busca por vantagens competitivas e da percepção dos profissionais de supply chain que o desempenho dos fornecedores não era satisfatório, o corpo gerencial da empresa

Persp. Online: exatas \& eng., Campos dos Goytacazes, 28 (10) 1 - 17 - 2020 
em que se realizou a pesquisa entendeu que a gestão de fornecedores seria o ponto central a ser melhorado. Essa decisão se deu após diversas discussões junto à equipe do setor de suprimentos e, especialmente, após a análise de indicadores de desempenho que serão apresentados nos próximos tópicos deste trabalho.

Portanto, na primeira etapa do método DMAIC, a organização definiu que o processo de gerenciamento de fornecedores seria revisto em busca de oportunidades de melhoria. Mais especificamente, os principais problemas a serem solucionados foram definidos como o elevado percentual de atraso nas entregas de materiais e a frequente ocorrência de não conformidades ao longo do processo de suprimentos.

\subsection{Medir}

$\mathrm{Na}$ etapa de medição foram utilizadas duas abordagens a fim de conseguir um panorama do atual gerenciamento de fornecedores: mapeamento de processos e cáculo de indicadores de desempenho. A primeira técnica empregada foi o mapeamento do processo, a representação dos subprocessos ou atividades que o compõem, de modo a tornar possível o seu entendimento. Na presente pesquisa foi utilizada uma representação simples por meio de fluxogramas, devido a sua fácil adaptação e simplicidade (ABPMP, 2013).

Mediante observação, conversas com os profissionais do setor e estudo dos procedimentos existentes na companhia chegou-se às etapas representadas na Figura 1. Esses são os subprocessos que compõe o processo de gestão de fornecedores realizado pelo setor de supply chain da empresa estudada.

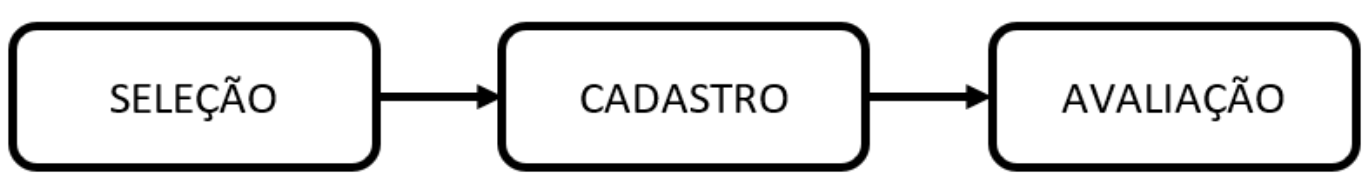

Figura 1: Etapas do processo original de gerenciamento de fornecedores utilizado na empresa.

A seleção do fornecedor fica a cargo do comprador. De posse da demanda pelo material ou serviço, cabe a ele buscar alternativas no mercado e fazer uma triagem dos possíveis fornecedores. São exigidos documentos básicos comprovando a regularidade das instalações do fornecedor e, caso aplicável, determinados documentos específicos (certificações, atestados de capacidade, etc.). Estando a empresa apta a fornecer, são encaminhados os documentos e dados cadastrais para o setor de cadastro, que realiza o registro no sistema ERP da organização. Os fornecedores cadastrados passam por uma avaliação anual na qual são avaliados qualitativamente pelos compradores por meio de um questionário, resultando em uma nota final que tem por objetivo expressar a qualidade no atendimento do fornecedor durante aquele ano.

Depois do mapeamento, a segunda técnica usada foi a medição de indicadores quantitativos que ilustram a eficiência do processo. Os indicadores escolhidos para aplicação nesta pesquisa tiveram como base a sugestão do Global Supply Chain Comittee (IADC, 2018), uma associação internacional composta pelas maiores empresas de perfuração existentes no mercado de petróleo, que visa estabelecer as melhores práticas para o setor em termos de gestão da cadeia de suprimentos. A escolha se baseia no fato de que tais indicadores são amplamente utilizados no mercado, o que facilita tanto a comunicação com fornecedores

Persp. Online: exatas \& eng., Campos dos Goytacazes, 28 (10) 1 - 17 - 2020 
quanto o intercâmbio de ideias entre as empresas de perfuração.

Dentre os indicadores sugeridos pela IADC (2018), foram escolhidos para serem aplicados na pesquisa: atraso na entrega de pedidos de compra, divergências no faturamento, não conformidades físicas e quantidades divergentes. Esses indicadores foram escolhidos pela sua relevância e influência no processo estudado, adaptação à estratégia de gestão da cadeia de suprimentos da empresa e adaptabilidade aos recursos disponíveis. Para uma melhor organização do artigo, os indicadores foram detalhados na etapa de Melhoria do método DMAIC.

A medição do período anterior à aplicação da metodologia, no segundo trimestre de 2017, apresentou os resultados mostrados na Tabela 1. Os problemas apontados pela organização, tais como atrasos de entrega e não conformidades frequentes, foram comprovados pelo cálculo dos indicadores.

Tabela 1: Resultados iniciais dos indicadores de desempenho calculados para os fornecedores da companhia.

\begin{tabular}{cc}
\hline Fornecedores avaliados & 575 \\
\hline Lead time total do processo de compra (dias) & 77,7 \\
\hline Indicador Atraso na entrega & $63,8 \%$ \\
\hline Indicador Divergências no faturamento & Sem dados \\
\hline Indicador Não Conformidades físicas & $0,75 \%$ \\
\hline Indicador Quantidade divergente & $0,01 \%$ \\
\hline
\end{tabular}

Como resultado desta etapa de medição do método DMAIC nota-se que o processo de gestão de fornecedores ainda se mostra incipiente. O processo é composto por apenas três etapas que geram pouco valor para a empresa e dificilmente trarão melhoria no desempenho dos fornecedores. Não conformidades ligadas à divergência no faturamento não eram registradas, por isso a ausência de dados.

\subsection{Analisar}

A partir da etapa de medição foi possível realizar uma análise sobre o desempenho do processo e evidenciar os pontos a serem melhorados. O detalhamento de cada problema é apresentado ao longo dos tópicos a seguir e as ações tomadas para solucioná-los serão vistas na próxima etapa do método DMAIC.

- Avaliações são feitas apenas pelos compradores, não levando em consideração a percepção dos usuários do produto.

Autores como Gunasekaran et al. (2004) sugerem que um processo de avaliação de fornecedores deve conter três níveis de critérios: estratégico, tático e operacional. A avaliação feita pelo setor de compras na empresa pesquisas abrange critérios estratégicos e táticos, como qualidade de comunicação, nível de preços em comparação ao mercado, flexibilidade e agilidade na resolução de não conformidades. Porém os critérios de nível operacional, como defeitos durante a utilização e assistência técnica, devem ser avaliados pelos usuários do material, o que não acontece.

Persp. Online: exatas \& eng., Campos dos Goytacazes, 28 (10) 1 - 17 - 2020 
- Falta de uma classificação dos fornecedores quanto a sua criticidade.

A ausência dessa classificação acaba por dificultar o gereciamento dos fornecedores e não permite que sejam adotadas estratégias distintas para cada grupo. O relacionamento com fornecedores críticos deve ser gerenciado com uma atenção especial pela organização.

- Ausência de uma etapa de qualificação, antes do cadastro, e uma etapa de requalificação periódica, a fim de garantir a qualidade das empresas cadastradas.

Uma etapa de qualificação realizada de forma correta garante à empresa que seus fornecedores estejam alinhados com sua estratégia e possuam os requisitos de qualidade exigidos. Já a etapa de requalificação faz com que esses critérios sejam revisitados e mantidos ao longo do tempo (KANNAN e TAN, 2002). O processo observado durante pesquisa não contempla essas etapas.

- A performance dos fornecedores não é medida por meio de indicadores de desempenho quantitativos que sejam refletidos na avaliação anual.

Como mencionado anteriormente, existe uma etapa de avaliação realizada pelos compradores, porém ela possui um caráter exclusivamente qualitativo. Não são utilizados indicadores de desempenho quantitativos na avaliação.

- Ausência de uma lista geral de fornecedores aprovados.

Possuir uma lista contendo todos os fornecedores aprovados disponíveis para utilização pode ser uma boa ferramenta para o dia-a-dia do setor de suprimentos, e de outros setores da empresa que enviam demandas ao setor de compras. Se utilizada como ferramenta de consulta, a lista ajuda os compradores a encontrar rapidamente os fornecedores cadastrados para determinado escopo de serviço ou a verificar com facilidade se determinado fornecedor é considerado crítico ou não.

- Processo de avaliação não resulta em melhoria de desempenho dos fornecedores. São frequentes problemas como atrasos na entrega e não conformidades, gerando desperdícios e custos que poderiam ser evitados.

A etapa de avaliação existente não se mostra eficiente em gerar uma melhor performance dos fornecedores. Apesar dos fornecedores serem comunicados a respeito das avaliações, os problemas de atraso na entrega e não conformidades permanecem.

Como o processo de gestão de fornecedores ainda não é percebido como plenamente desenvolvido, o efeito das avaliações não é o desejado.

- Número de fornecedores considerado elevado para as necessidades da empresa.

Por meio do sistema ERP da empresa foi possível extrair a informação de que havia muitos fornecedores cadastrados sem serem utilizados para nenhuma aquisição. Para a implementação de um sistema robusto de gestão de fornecedores e medição de desempenho é necessário que a base de fornecedores seja reduzida, a fim de tornar possível um gerenciamento eficaz. Essa prática vai de encontro ao sugerido pela metodologia lean, que prega um relacionamento mais estreito com uma base reduzida de fornecedores (BHATTACHARYA et al., 1996).

Persp. Online: exatas \& eng., Campos dos Goytacazes, 28 (10) 1 - 17 - 2020 


\subsection{Melhorar}

Tendo em mãos a definição do problema e os resultados da fase de análise anterior foi possível colocar em prática as melhorias necessárias.

A primeira decisão foi definir níveis diferentes de criticidade para os fornecedores da companhia, o que permitiu à empresa otimizar os recursos empregados na gestão de fornecedores, gerenciando mais de perto aqueles considerados críticos. Essa mudança vai ao encontro ao trabalho de Dyer et al. (1998), que sugerem a definição de critérios distintos para tipos diferentes de fornecedores.

Nesta pesquisa, para realizar a definição dos fornecedores críticos, foi utilizada a "Matriz" (ou "Modelo") de Kraljic (1983), que leva em consideração dois fatores na estratégia de compras de uma empresa: o impacto financeiro (tão maior quanto maior for o dispêndio financeiro da companhia com determinado material ou serviço) e o risco de fornecimento (tão maior quanto menor for o número de opções de fornecedores no mercado), sendo esses os eixos da matriz. Cada quadrante da matriz apresenta uma classificação diferente de produtos e serviços, e cada um exige estratégias diferentes na gestão de fornecedores.

Existem diversas adaptações da Matriz de Kraljic, dependendo das necessidades da organização que a utiliza e do segmento de mercado no qual ela está inserida. Para o caso em estudo, foram estabelecidos os critérios para definição de criticidade como sendo: o risco de fornecimento e o impacto do material ou serviço na qualidade da operação de perfuração da empresa e/ou nas áreas de segurança, meio ambiente e saúde. A matriz obtida é apresentada na Figura 2.

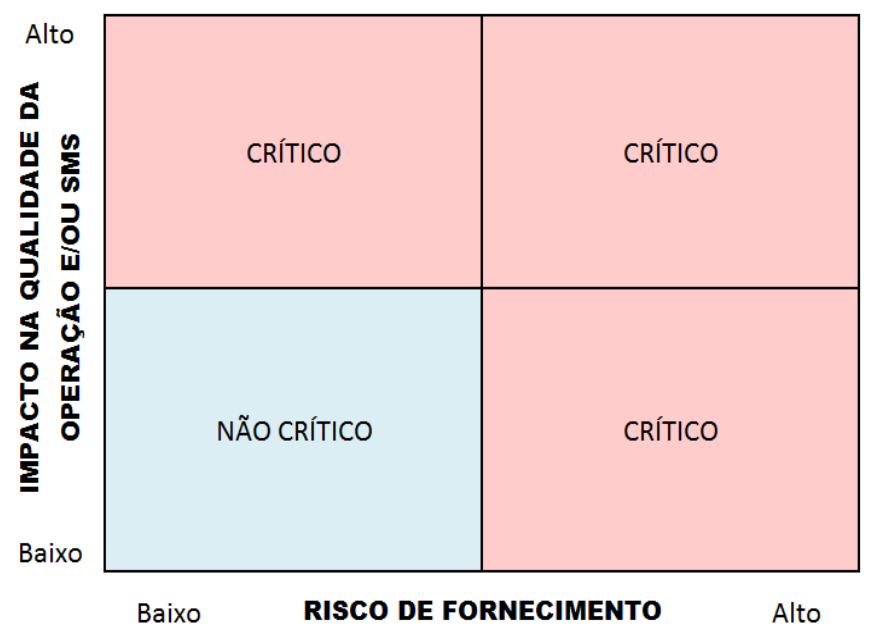

Figura 2: Matriz de Kraljic adaptada para definição de fornecedores críticos.

A Figura 2 passa a falsa impressão de que a grande maioria dos fornecedores se enquadra como crítico, quando na verdade apenas $18 \%$ do banco de fornecedores foi considerado crítico, dado que apenas uma pequena parte do total abastece a empresa com produtos e serviços de alto impacto e/ou risco de fornecimento.

Em um segundo momento, houve a inclusão de uma etapa robusta de qualificação que deve preceder o cadastro de qualquer nova empresa considerada crítica no banco de fornecedores. A seleção de possíveis fornecedores continua a cargo do comprador, porém

Persp. Online: exatas \& eng., Campos dos Goytacazes, 28 (10) 1 - 17 - 2020 
para todo fornecedor crítico deve haver uma auditoria in loco para verificar critérios de qualidade, saúde, segurança e meio ambiente. Os critérios foram definidos em conjunto com as respectivas áreas técnicas, sempre visando alinhar a qualificação dos fornecedores com os requisitos da companhia.

Para complementar a avaliação qualitativa que era feita periodicamente estabeleceuse o cálculo de quatro indicadores de desempenho para os fornecedores de materiais (dado que essa avaliação quantitativa não se aplica aos fornecedores de serviço). A avaliação periódica pode englobar uma série de critérios diferentes. Cabe à empresa definir os mais relevantes para o seu negócio. O desafio é combinar os critérios corretos, incluindo fatores qualitativos e quantitativos, tendo como resultado um índice final que possa mostrar de forma clara o desempenho de determinado fornecedor.

Os indicadores de desempenho são calculados a cada três meses. A definição da periodicidade se deu por intermédio de uma análise do histórico de compras da empresa, mais especificamente do tempo de entrega dos pedidos (da criação do pedido até o recebimento e conferência). Nos últimos doze meses aproximadamente $90 \%$ dos pedidos tiveram seus tempos de entrega menores que três meses, portanto o cálculo de indicadores utilizando esse período traz uma boa representação, uma vez que é possível cobrir a maioria dos pedidos de compra em seu ciclo completo.

Os resultados obtidos são compilados anualmente, juntamente com o questionário qualitativo. Para definição de quais índices seriam medidos utilizou-se como base as métricas sugeridas pelo Global Supply Chain Comittee (IADC, 2018). Os indicadores foram definidos da seguinte forma:

$$
\begin{gathered}
\% \text { Atraso na entrega }=\frac{\text { Total de itens entregues fora do prazo }}{\text { Total de itens recebidos }} \\
\% \text { Divergências no faturamento }=\frac{\text { Total de itens faturados incorretamente }}{\text { Total de itens recebidos }} \\
\% \text { Não conformidade física }=\frac{\text { Total de itens danificados }}{\text { Total de itens recebidos }} \\
\% \text { Quantidade divergente }=\frac{\text { Total de itens com quantidades divergentes }}{\text { Total de itens recebidos }}
\end{gathered}
$$

Os dados usados nos indicadores são obtidos do sistema ERP da empresa, e o seu cálculo é realizado por meio do software Microsoft Excel. A empresa espera incorporar esse cálculo ao sistema ERP em breve.

Com objetivo de monitorar a qualificação dos fornecedores apresentada antes do cadastro foi implementada a etapa de requalificação ao processo. Os fornecedores críticos são requalificados a cada três anos, enquanto os não-críticos passam pelo processo a cada cinco anos. O objetivo é verificar se os critérios verificados na qualificação da empresa continuam sendo cumpridos. Os tempos de requalificação foram definidos com base nas

Persp. Online: exatas \& eng., Campos dos Goytacazes, 28 (10) 1 - 17 - 2020 
validades dos documentos e certificações que os fornecedores precisam apresentar no ato da qualificação.

A fim de acrescentar a percepção dos usuários dos materiais ou serviços ao processo de avaliação, os colaboradores de áreas operacionais da empresa e a bordo das plataformas offshore passaram a também avaliar os fornecedores mediante questionários. Essas avaliações, assim como as dos compradores, são compiladas anualmente e integram a nota final do fornecedor, possibilitando que a percepção de todos os participantes seja levada em consideração.

O fluxograma do processo após as mudanças é apresentado na Figura 3.

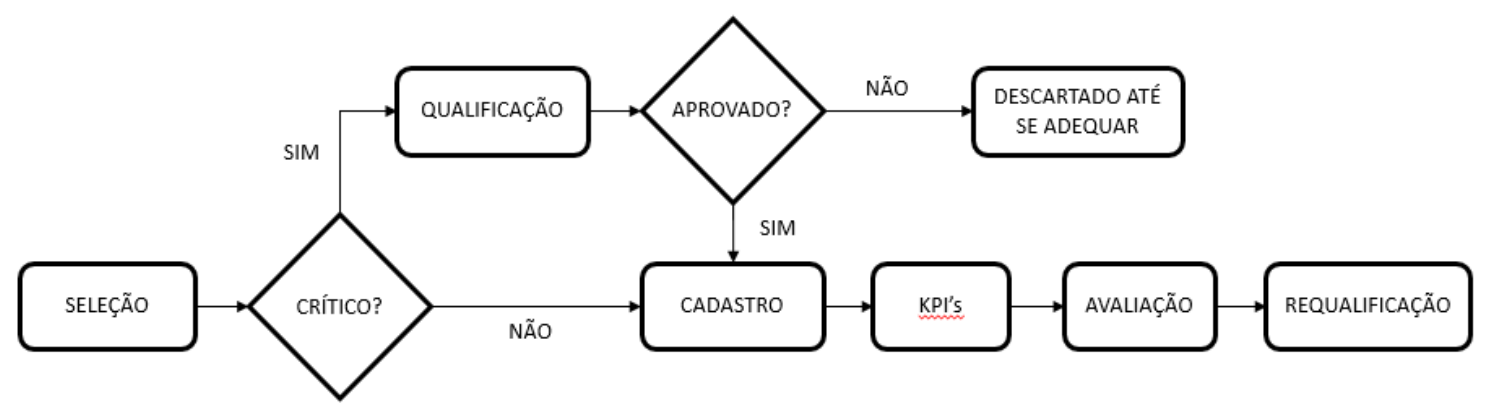

Figura 3: Etapas do processo de gerenciamento de fornecedores após as modificações.

Com essas medidas, espera-se controlar o processo remodelado de maneira eficiente ao longo do tempo. As alterações foram refletidas nos procedimentos da companhia e todos os colaboradores foram treinados por meio de apresentações feitas pelo setor de supply chain.

\subsection{Controlar}

O processo remodelado por intermédio do DMAIC e com base nas melhores práticas indicadas pela literatura sobre gerenciamento de fornecedores na cadeia de suprimentos trouxe benefícios perceptíveis à organização.

A Tabela 2 ilustra o andamento do projeto dividido por trimestres, partindo da primeira medição de resultados, no segundo trimestre de 2017.

Nota-se uma grande diminuição (cerca de 54\%) no número de fornecedores. Trata-se do reflexo de um processo de qualificação mais rigoroso e no bloqueio daqueles com baixa performance ao longo do tempo. Tornando a base de fornecedor mais enxuta reduziu-se o desperdício de recursos no controle daqueles que eram pouco usados ou que não atendiam às exigências da empresa, permitindo um controle mais eficiente daqueles que permaneceram, especialmente os classificados como críticos.

Tabela 2: Resultados obtidos com a implementação da nova metodologia de gestão de fornecedores no decorrer do tempo.

\begin{tabular}{ccccccc}
\hline \multicolumn{8}{c}{ Período } \\
\hline $\mathbf{1}$ & $\mathbf{2}$ & $\mathbf{3}$ & $\mathbf{4}$ & 5 & 6 \\
\hline
\end{tabular}

Persp. Online: exatas \& eng., Campos dos Goytacazes, 28 (10) 1 - 17 - 2020 


\begin{tabular}{ccccccc}
\hline $\begin{array}{c}\text { Fornecedores } \\
\text { avaliados }\end{array}$ & 575 & 575 & 373 & 381 & 336 & 264 \\
\hline $\begin{array}{c}\text { Lead time total } \\
\text { do processo de } \\
\text { compra (dias) }\end{array}$ & 77,7 & 79,7 & 60,4 & 54,8 & 51,7 & 35,5 \\
\hline $\begin{array}{c}\text { Indicador } \\
\text { Atraso na } \\
\text { entrega }\end{array}$ & $63,8 \%$ & $39,3 \%$ & $36,6 \%$ & $31,6 \%$ & $40,2 \%$ & $38,6 \%$ \\
\hline $\begin{array}{c}\text { Indicador } \\
\text { Divergências no } \\
\text { faturamento }\end{array}$ & Sem dados & $0,05 \%$ & $0,05 \%$ & $0,03 \%$ & $0,06 \%$ & $0,03 \%$ \\
\hline $\begin{array}{c}\text { Indicador Não } \\
\text { Conformidades } \\
\text { físicas }\end{array}$ & $0,75 \%$ & $0,82 \%$ & $0,43 \%$ & $0,25 \%$ & $0,39 \%$ & $0,26 \%$ \\
\hline $\begin{array}{c}\text { Indicador } \\
\text { Quantidade } \\
\text { divergente }\end{array}$ & $0,01 \%$ & $0,03 \%$ & $0,04 \%$ & $0,02 \%$ & $0,06 \%$ & $0,06 \%$ \\
\hline
\end{tabular}

O lead time do processo de compra é formado por uma série de intervalos menores que compõe o tempo total, entre eles: o tempo de resposta da cotação pelo fornecedor, o tempo gasto pelo comprador para fechar o pedido de compra e o tempo gasto pelo fornecedor para entregar o pedido que foi colocado.

O tempo total caiu mais do que $50 \%$, evidenciando um considerável aumento na eficiência do processo. Com uma sistemática bem definida de avaliação de desempenho, que inclui o feedback para o fornecedor a respeito de sua performance, a tendência é que o senso de competição aumente no mercado, possibilitando que as cotações sejam respondidas mais rapidamente e haja um esforço para diminuir prazos de entrega dos pedidos. Além disso, com uma base robusta na qual apoiar as decisões, os compradores conseguem diminuir seu tempo de análise para fechar os pedidos de compra, também contribuindo para um menor tempo total.

Os indicadores de desempenho precisam ser analisados separadamente para um melhor entendimento. Nota-se que o percentual de itens entregues atrasados sofreu uma diminuição significativa, saindo de $63,8 \%$ para $38,6 \%$ no último período. Essa melhoria tem um reflexo direto lead time total do processo mencionado no parágrafo anterior. Apresentando um comportamento semelhante, o indicador de não conformidades físicas sofreu uma queda considerável, de $0,75 \%$ para $0,26 \%$ no último cálculo realizado, evidenciando uma melhoria considerável no desempenho dos fornecedores neste quesito.

Já os indicadores de divergências no faturamento e nas quantidades entregues não tiveram a mesma tendência. O primeiro apresentou uma diminuição pouco expressiva, enquanto o segundo sofreu um aumento considerável, evidenciando uma queda de qualidade dos fornecedores neste item. Após analisar internamente a empresa entende-se que esses indicadores não foram trabalhados junto aos fornecedores tanto quanto os demais. Esse fato serviu como aprendizado para que o processo seja melhorado daqui em diante.

\section{CONCLUSÕES}

Persp. Online: exatas \& eng., Campos dos Goytacazes, 28 (10) 1 - 17 - 2020

https://ojs3.perspectivasonline.com.br/ 
Conclui-se que, utilizando os princípios da metodologia Lean Six Sigma, é possível melhorar a qualidade de um sistema de gerenciamento de fornecedores, além de tornar mais eficiente a cadeia de suprimentos de uma organização, mesmo tratando-se de um mercado complexo como o de óleo e gás.

A ferramenta DMAIC se mostrou muito eficiente ao ser aplicada no problema em questão. A estruturação das etapas sugerida pelo DMAIC foi essencial para organização do projeto e fundamentação das melhorias sugeridas para a empresa que foi palco da pesquisa. Mais do que isso, o sucesso desta aplicação sugere um alinhamento positivo entre o lean manufacturing e a gestão da cadeia de suprimentos. As diretrizes do pensamento lean vão ao encontro da eficiência perseguida pelo supply chain management, especialmente em cenários competitivos como o atual mercado de óleo e gás.

Os objetivos iniciais da pesquisa foram atingidos. A maioria dos indicadores de desempenho apresentou uma melhora significativa, como é possível verificar na seção anterior. $\mathrm{O}$ percentual de atrasos na entrega e o número de não conformidades, que eram os focos do projeto, foram substancialmente reduzidos.

Essas melhorias vêm trazendo impactos significativos para as operações da companhia. Os profissionais da empresa que dependem dos materiais comprados em seu diaa-dia conseguem se programar melhor ao realizar uma requisição de compra; a empresa evita os custos que uma possível falta de material poderia trazer; os compradores melhoram consideravelmente o planejamento de suas atividades, pois gastam menos tempo na resolução de não conformidades e atrasos; e o trabalho do setor de suprimentos se torna mais estratégico dentro da organização.

Uma possível pesquisa futura pode ir adiante com a quantificação da redução de custos advinda das mudanças realizadas, de modo a evidenciar o consequente impacto nos lucros da empresa a partir de um processo estruturado de gestão de fornecedores.

\section{REFERÊNCIAS}

ABPMP. ASSOCIATION OF BUSINESS PROCESS MANAGEMENT PROFESSIONALS. Guia para o Gerenciamento de Processos de Negócio: BPM CBOK V3.0. 2013. Disponível em: <https://cdn.ymaws.com/www.abpmp.org/resource/resmgr/Docs/ABPMP_CBOK_Guide_ Portuguese.pdf >. Acesso em: 22 ago. 2019

ALBLIWI, S. A.; ANTONY, J.; LIM, S. A. halim. A systematic review of Lean Six Sigma for the manufacturing industry. Business Process Management Journal, vol. 21, n. 3, p. 665-691. 2015.

ANDERSEN, M; SKJOETT-LARSEN, T. Corporate social responsibility in global supply chains. Supply Chain Management: An International Journal, v. 14, n. 2, p. 75-86, 2009.

BARGERSTOCK, A.S.; RICHARDS, S.R. Application of DMAIC to Academic Assessment in Higher Education. Quality Approaches in Higher education, v. 6, n.2., p. 31-40, 2015.

Persp. Online: exatas \& eng., Campos dos Goytacazes, 28 (10) 1 - 17 - 2020 
BHATTACHARYA, A. K.; COLEMAN, J. L.; BRACE, G.; KELLY, P. J. The Structure Conundrum in Supply Chain Management. The International Journal of Logistics Management, v. 7, n. 1, p. 39-48. 1996.

BOWERSOX, Donald J.; et al. Supply Chain Logistics Management. 4.ed. New York: McGraw-Hill. 2013.

BRITO, Renata; BERARDI, Patricia. Vantagem competitiva na gestão sustentável da cadeia de suprimentos: um metaestudo, Revista de Administração de Empresas. Fundação Getulio Vargas, Escola de Administração de Empresas de S.Paulo, v. 50, n. 2, p. 155-169, 2010.

DHAFER, Abdulla Y.; ARAMCO, Saudi. Introduction of Lean Six Sigma principles into plant paintenance work order cycle time. Proceedings of the 2012 International Conference on Industrial Engineering and Operations Management; 2012 Jul 3-6; Istanbul, Turquia. 2012.

DREACHSLIN, J.; LEE, P. D. Applying Six Sigma and DMAIC to diversity initiatives. Journal of healthcare management, v. 52, n. 6, p. 361-367, 2007.

DRESCH, A.; LACERDA, D. P.; JÚNIOR, J. A. V. A. Design Science Research: método de pesquisa para avanço da ciência e tecnologia. 1.ed. Porto Alegre: Bookman. 2015.

DYER, Jeffrey H. Specialized supplier networks as a source of competitive advantage: evidence from the auto industry. Strategic Management Journal, v. 17, n. 4, p. 271-291, 1996.

DYER, Jeffrey H.; CHO, D. S.; CHU, W. Strategic Supplier Segmentation: The Next "Best Practice" in Supply Chain Management. California Management Review, v. 40, n. 2, p. 5777. 1998.

FIRAT, S. Ü. O.; AKAN, M. Ö. A.; ERSOY, E.; GÖK, S.; ÜNAL, U. A Six Sigma DMAIC Process for Supplier Performance Evaluation using AHP and Kano's Model. International Journal of Business Analytics, v. 4, n. 2, p. 37-61. 2017.

FONSECA, João José Saraiva da. Metodologia da Pesquisa Científica. Fortaleza: Universidade Estadual do Ceará, 2002. (Apostila).

GIL, A. C. Como classificar as pesquisas. In: GIL, A. C. Como elaborar projetos de pesquisa. 4a ed. São Paulo: Atlas; 2002.

GUNASEKARAN, A.; PATELB, C.; MCGAUGHEYC, Ronald E. A framework for supply chain performance measurement. Int. J. Production Economics, v. 87, p. 333-347. 2004.

HOINASKI, F. Gestão de fornecedores: por que adotar esse processo. 2016. Disponível em: <https://www.ibid.com.br/blog/gestao-de-fornecedores>. Acesso em: 13 abr. 2019.

IADC, Global Supply Chain Comittee. Supplier Performance Metrics and Scorecard (GU-IADC-SC-002) Rev. 1. 2018. Disponível em: <http://www.iadc.org/wpcontent/uploads/2018/04/IADC-SUPPLIER-PERFORMANCE-REV-1-FINAL-9-APRIL2018.pdf >. Acesso em: 14 abr. 2019.

Persp. Online: exatas \& eng., Campos dos Goytacazes, 28 (10) 1 - $17-2020$ https://ojs3.perspectivasonline.com.br/ 
KANNAN, V. R.; TAN, K. C. Supplier Selection and Assessment: Their Impact on Business Performance. The Journal of Supply Chain Management, v. 38, n. 4, p. 11-21. 2002.

KRALJIC, P. Purchasing Must Become Supply Management. Harvard Business Review, p. 109-117. Set. 1983.

LUMMUS, R. R.; VOKURKA, R. J. Defining supply chain management: a historical perspective and practical guidelines. Industrial Management \& Data Systems, v. 99, n. 1, p. 11-17. 1999.

MARTINS, F. C. Gestão da Cadeia de Suprimentos: Aprimoramento da Metodologia de Diagnóstico e Proposição de um Método para Implantação baseado em Processos de Negócio. Congresso de Iniciação Científica UNIMEP, Piracicaba. Nov. 2011.

PARK, J.; SHIN, K.; CHANG, T.; PARK, J. An integrative framework for supplier relationship management. Industrial Management \& Data Systems, v. 110, n. 4, p. 495515. 2010.

REHMAN, S. T.; KHAN, S. A.; KUSI-SARPONG, S.; HASSAN, S. M. Supply chain performance measurement and improvement system. Journal of Modelling in Management, v. 13, n. 3, p. 522-549. 2018.

RODRIGUES D. M.; SELLITTO, M. A. Análise do desempenho de fornecedores de uma empresa de manufatura apoiada em análise de aglomerados. Produção, v. 19, n. 1, p. 055-069. 2009.

SALAH, Souraj et al. Implementation of Lean Six Sigma (LSS) in supply chain management (SCM): an integrated management philosophy. International Journal of Transitions and Innovation Systems, v. 1, p. 138-162. Jan. 2011.

SALAH, S.; RAHIM, A.; CARRETERO, J. A. The integration of Six Sigma and lean management. International Journal of Lean Six Sigma, v. 1, n. 3, p. 249-274. 2010.

SCHOLTEN, K.; SCHILDER, S. The role of collaboration in supply chain resilience. Supply Chain Management: An International Journal, v. 20, n. 4, p. 471-484. 2015.

SILVEIRA, D. T.; CÓRDOVA, F. P. Unidade 2 - A pesquisa científica. Métodos de pesquisa. Porto Alegre: UFRGS, p. 31-42, 2009.

SOKOVIC, M. et al. Quality Improvement Methodologies - PDCA Cycle, RADAR Matrix, DMAIC and DFSS. Journal of Achievements in Materials and Manufacturing Engineering, v. 43, n. 1, p. 476-483. Nov. 2010.

TAHERDOOST, H.; BRARD, A. Analyzing the Process of Supplier Selection Criteria and Methods. Procedia Manufacturing, v. 32, p. 1024-1034. 2019.

USUI, T.; KOTABE, M.; MURRAY, J. Y. A Dynamic Process of Building Global Supply Chain Competence by New Ventures: The Case of Uniqlo. Journal of International Marketing, v. 25, n. 3, p. 1-20. 2017.

Persp. Online: exatas \& eng., Campos dos Goytacazes, 28 (10) 1 - 17 - 2020 